\title{
MAXIMIZAÇÃO DA PRODUÇÃO E DA RECEITA AGRÍCOLA COM LIMITAÇÕES DE ÁGUA E NITROGÊNIO UTILIZANDO MÉTODO DE PONTOS INTERIORES
}

\author{
DANIEL F. DE CARVALHO ${ }^{1}$, ANGEL R. SANCHEZ DELGADO ${ }^{2}$, \\ ROSANE F. DE OLIVEIRA ${ }^{2}$, WILSON A. DA SILVA ${ }^{3}$, VINICIUS L. DO FORTE ${ }^{4}$
}

RESUMO: Com este trabalho, teve-se o objetivo de pesquisar o desempenho do método de pontos interiores (MPI), tipo trajetória central, na maximização de funções-objetivo associadas à produção e à receita agrícolas, com restrições de água e de nitrogênio. Para isso, foi desenvolvido um procedimento matemático que maximiza a produção e a receita líquida separadamente, em função da água e do nitrogênio aplicados à cultura numa matriz bidimensional de restrições, a partir de funções de produtividade para as culturas da aveia e da laranja-pera. O modelo foi caracterizado e, a partir dele, foram gerados resultados numéricos para diferentes cenários agrícolas. Foi possível concluir que a metodologia utilizada (MPI) foi capaz de maximizar as funções de produtividade e de receita, resultando na obtenção de valores semelhantes aos publicados na literatura, utilizando-se de programação separável.

PALAVRAS-CHAVE: programação não linear, trajetória central, concavidade, função-resposta, otimização.

\section{MAXIMIZATION OF THE PRODUCTION AND AGRICULTURAL INCOME WITH WATER AND NITROGEN CONSTRAINTS BY USING THE INTERIOR POINTS METHOD}

\begin{abstract}
The objective of this work was to evaluate the interior point method (central path) (IPM) in the maximization of objective functions associated with the agricultural production and income with water and nitrogen constraints. For this purpose, a procedure to maximize the production and income in relation to water and nitrogen applied to the culture was developed in a bi-dimensional matrix of constraints. The model was characterized and the numerical results are presented for different agricultural crops and scenes. It was concluded that the used methodology was capable of maximizing the productivity, obtaining values similar to the ones published in literature by using separable programming.
\end{abstract}

KEYWORDS: nonlinear programming, central path, concavity, response function, optimization.

\section{INTRODUÇÃO}

A exploração de qualquer atividade agrícola que se destina à obtenção de um produto requer a utilização de insumos, que são combinados em quantidade e em qualidade por quem decide realizar a exploração, conforme o conhecimento de tecnologias disponíveis. A relação entre o processo de conversão de diversos fatores de produção (insumos) em determinado produto caracteriza uma função de produção, cuja relação insumo-produto pode ser contínua ou descontínua (AGUIAR, 2005).

Vários fatores referentes ao solo, à planta e à atmosfera interagem entre si, determinando a produtividade das culturas agrícolas. Certamente existe uma relação funcional entre esses fatores e a produção das culturas, características de cada condição ambiental. A resposta das culturas à irrigação pode variar em diferentes tipos de solos, climas e também em decorrência da quantidade e

\footnotetext{
${ }^{1}$ Professor Associado, Departamento de Engenharia, UFRRJ, Seropédica - RJ, carvalho@ufrrj.br. Bolsista do CNPq.

${ }^{2}$ Professor Associado, Departamento de Matemática, UFRRJ, Seropédica - RJ, asanchez@ufrrj.br

${ }^{3}$ Professor Adjunto, Departamento de Ciências Agrárias, UEMA/CESI, Imperatriz - MA.

${ }^{4}$ Bacharel em Matemática Aplicada e Computacional pela UFRRJ, Mestrando em Engenharia de Sistemas e Computação na UFRJ.

Recebido pelo Conselho Editorial em: 11-12-2007

Aprovado pelo Conselho Editorial em: 11-5-2009
} 
da frequência de aplicação de água. O efeito da água na produção pode ainda interagir com fertilizantes e uma série de outros insumos (FRIZZONE \& ANDRADE JÚNIOR, 2005).

O manejo racional dos insumos é imperativo na otimização da produção agrícola. A avaliação econômica da produção envolve a quantificação da produtividade em resposta ao total de insumos aplicados. A água e o nitrogênio são fundamentais ao desenvolvimento das culturas, e quando são correlacionadas à produção obtida, tem-se a função de produção água-nitrogênio-cultura.

O emprego das funções de produção na análise dos resultados de experiências agrícolas é bastante difundido (FRIZZONE et al., 1995; BERTONHA et al., 1999; PEREIRA et al., 2003; FRIZZONE \& ANDRADE JÚNIOR, 2005). Diversos trabalhos mostram que, para a função de produção água-fertilizante-cultura, um modelo polinomial de segundo grau normalmente é utilizado. Essas funções não lineares podem ser tratadas por aproximações lineares, e a sua otimização pode ser feita utilizando-se da técnica de programação separável. Essa abordagem permite transformar um problema de programação não linear em um problema de programação linear, possível de ser solucionado pelo Método Simplex. Conforme FRIZZONE \& ANDRADE JÚNIOR (2005), essa técnica permite aproximar uma função não linear por meio de um conjunto de segmentos de retas. De acordo com os autores, o desenvolvimento desse tipo de programação inicia-se por um equacionamento cujo esquema permite aproximar, por partes, funções não lineares a funções lineares. A programação linear e a programação quadrática são, sem dúvida, apropriadas para problemas complexos que não podem ser resolvidos satisfatoriamente com técnicas analíticas convencionais (BAIO et al., 2004; OJIMA YAMAKAMI, 2006).

Desenvolvida a partir do trabalho de KARMARKAR (1984), a metodologia de pontos interiores (MPI), tipo trajetória central, tem apresentado resultados com maior confiabilidade quando aplicada em procedimentos que trabalham diretamente com as funções-objetivo. Segundo BARBOZA \& OLIVEIRA (2006), uma vantagem para o uso dessa metodologia está na montagem de estrutura matricial bem definida do problema. Experiências anteriores com o desenvolvimento de MPIs específicos para uma classe de problemas e a exploração da estrutura matricial dos sistemas lineares resultantes mostram que essa abordagem é muito bem-sucedida em termos da obtenção de melhor desempenho computacional.

Com base no exposto, desenvolveu-se este trabalho com o objetivo de implementar procedimento baseado no MPI, tipo trajetória central, como alternativa à técnica de programação separável (FRIZZONE \& ANDRADE JÚNIOR, 2005). A fim de avaliar o desempenho metodológico, é apresentada uma experiência numérica realizada com base nos dados apresentados por FRIZZONE et al. (1995) e BERTONHA et al. (1999).

\section{MATERIAL E MÉTODOS}

Seja $p(w, n)$ a função-resposta de uma determinada cultura ( $\mathrm{t} \mathrm{ha}^{-1}$ ou caixa árvore $\left.{ }^{-1}\right)$ em relação à lâmina de água $w\left(\mathrm{~mm}\right.$ ou $\mathrm{L}$ árvore $\left.{ }^{-1}\right)$ e à dose de fertilizantes $n\left(\mathrm{~kg} \mathrm{ha}^{-1}\right.$ ou $\mathrm{kg}$ árvore $\left.{ }^{-1}\right)$, em geral uma função não linear, e $w_{l}, w_{u}, n_{l}, n_{u}$ limitantes inferiores e superiores de $w$ e $n$, respectivamente, sendo $w_{l} \geq 0, n_{l} \geq 0, w_{u} \geq w_{l}, n_{u} \geq n_{l}$.

Nesse contexto, o primeiro problema a considerar é: maximizar $p(w, n)$; sujeito a $w_{l} \leq w \leq w_{u}, n_{l} \leq n \leq n_{u}$. O problema caracteriza-se como sendo de programação não linear (PPNL) na caixa bidimensional de limitações $\left[w_{l}, w_{u}\right] x\left[n_{l}, n_{u}\right]$ (BERTSEKAS, 2004).

Outro problema interessante de estudar na otimização agrícola com limitações hídricas e de fertilizantes é a maximização da receita líquida obtida no plantio de uma determinada cultura. Considerando que o beneficio é proporcional à produtividade, procura-se, então: maximizar $R L(w, n)=p_{c} p(w, n)-c_{w} w-c_{n} n$; sujeito a $w_{l} \leq w \leq w_{u}, n_{l} \leq n \leq n_{u}$; em que $\mathrm{p}_{\mathrm{c}}$ representa o 
preço da cultura $\left(R \$ \mathrm{t}^{-1}\right), \mathrm{c}_{\mathrm{w}}$ o custo de água $\left(\mathrm{R} \$ \mathrm{~mm}^{-1} \mathrm{ha}^{-1}\right), \mathrm{c}_{\mathrm{n}}$ o custo do nitrogênio $\left(\mathrm{R} \$ \mathrm{~kg}^{-1}\right)$ e $R L(w, n)$ a receita líquida obtida do plantio $\left(\mathrm{R} \$ \mathrm{ha}^{-1}\right)$.

Considere o PPNL na forma quadrática: maximizar $\mathrm{p}(\mathrm{w}, \mathrm{n})$; sujeito a $w_{l} \leq w \leq w_{u}$, $n_{l} \leq n \leq n_{u} ; \mathrm{p}(\mathrm{w}, \mathrm{n})=\mathrm{aw}^{2}+\mathrm{bwn}+\mathrm{cn}^{2}+\mathrm{dw}+\mathrm{en}+\mathrm{f}$, sendo a, $\leq 0$. Conceitualmente, o MPI tipo trajetória central funciona da seguinte maneira: fixado um parâmetro $\mu>0$ e incorporando as restrições, que definem a caixa bidimensional na função-objetivo por meio de uma função "barreira logarítmica", resolve-se o PPNL irrestrito:

$$
\text { Maximizar } \phi_{\mu}(\mathrm{w}, \mathrm{n})
$$

em que,

$$
\begin{aligned}
& \phi_{\mu}(\mathrm{w}, \mathrm{n})=\mathrm{p}(\mathrm{w}, \mathrm{n})+\mu \mathrm{B}(\mathrm{w}, \mathrm{n}), \mathrm{e} \\
& \mathrm{B}(\mathrm{w}, \mathrm{n})=\log \left(\mathrm{w}_{\mathrm{u}}-\mathrm{w}\right)+\log \left(\mathrm{w}-\mathrm{w}_{1}\right)+\log \left(\mathrm{n}_{\mathrm{u}}-\mathrm{n}\right)+\log \left(\mathrm{n}-\mathrm{n}_{1}\right)
\end{aligned}
$$

Em seguida, faz-se decréscimo de $\mu$ e o processo é repetido até que um critério de parada seja satisfeito. O nome barreira logarítmica deve-se ao fato de que a função logaritmo obriga o procedimento a gerar pontos interiores afastados da fronteira da caixa bidimensional de restrições. Para cada $\mu$, o máximo de $\phi_{\mu}$ é alcançado num ponto interior do conjunto de soluções viáveis do problema, e quando $\mu$ tende a zero, esse ponto move-se até um ponto próximo da solução ótima do problema. Como função de $\mu$, o conjunto de soluções ótimas dos PPNLs irrestritos fornece uma curva denominada trajetória central. O importante da metodologia é a maximização de $\phi_{\mu}(\mathrm{w}, \mathrm{n})$ para $\mu$ fixo, mas como $\phi_{\mu}$ é uma função estritamente côncava, pelas condições de primeira ordem (BERTSEKAS, 2004), $(w, n)=(w(\mu), n(\mu))$ define uma solução ótima do PPNL irrestrito se, e somente se:

$$
\begin{aligned}
& \frac{\partial \varphi_{\mu}(\mathrm{w}, \mathrm{n})}{\partial \mathrm{w}}=\frac{\partial \mathrm{p}(\mathrm{w}, \mathrm{n})}{\partial \mathrm{w}}-\frac{\mu}{\mathrm{w}_{\mathrm{u}}-\mathrm{w}}+\frac{\mu}{\mathrm{w}-\mathrm{w}_{1}}=2 \mathrm{aw}+\mathrm{bn}+\mathrm{d}-\frac{\mu}{\mathrm{w}_{\mathrm{u}}-\mathrm{w}}+\frac{\mu}{\mathrm{w}-\mathrm{w}_{1}}=0 \\
& \frac{\partial \varphi_{\mu}(\mathrm{w}, \mathrm{n})}{\partial \mathrm{n}}=\frac{\partial \mathrm{p}(\mathrm{w}, \mathrm{n})}{\partial \mathrm{n}}-\frac{\mu}{\mathrm{n}_{\mathrm{u}}-\mathrm{n}}+\frac{\mu}{\mathrm{n}-\mathrm{n}_{1}}=2 \mathrm{cn}+\mathrm{bw}+\mathrm{e}-\frac{\mu}{\mathrm{n}_{\mathrm{u}}-\mathrm{n}}+\frac{\mu}{\mathrm{n}-\mathrm{n}_{1}}=0
\end{aligned}
$$

Fazendo $\quad s_{u}=\frac{\mu}{w_{u}-w}>0_{j} s_{l}=\frac{\mu}{\mathrm{w}-w_{l}}>0 ; z_{u}=\frac{\mu}{n_{u}-n}>0 ; z_{l}=\frac{\mu}{n-n_{l}}>0$, o sistema (4)-(5) pode ser escrito como o sistema não linear:

$$
\begin{aligned}
& 2 \mathrm{aw}+\mathrm{bn}+\mathrm{s}_{1}-\mathrm{s}_{\mathrm{u}}=-\mathrm{d} \\
& 2 \mathrm{cn}+\mathrm{bw}+\mathrm{z}_{1}-\mathrm{z}_{\mathrm{u}}=-\mathrm{e} \\
& \mathrm{s}_{\mathrm{u}}\left(\mathrm{w}_{\mathrm{u}}-\mathrm{w}\right)=\mathrm{u} \\
& \mathrm{s}_{1}\left(\mathrm{w}-\mathrm{w}_{1}\right)=\mathrm{u} \\
& \mathrm{z}_{\mathrm{u}}\left(\mathrm{n}_{\mathrm{u}}-\mathrm{n}\right)=\mathrm{u} \\
& \mathrm{z}_{1}\left(\mathrm{n}-\mathrm{n}_{1}\right)=\mathrm{u}
\end{aligned}
$$

Os pontos que resolvem aproximadamente as eqs.(6)-(11) encontram-se próximos da trajetória central associada à produtividade; mais ainda, as eqs.(6)-(7) representam as restrições que definem a região de viabilidade do correspondente problema dual, enquanto as eqs.(8)-(11) representam as condições de "folgas complementares aproximadas". Entre as vantagens das soluções duais, destacam-se as possibilidades de fornecer informações econômicas sobre a 
utilização de recursos, como, por exemplo, auxiliar na tomada de decisão para a aquisição de recursos adicionais ou na análise de sensitividade. Neste caso, as variáveis $s_{u^{\prime}} s_{v} z_{w^{\prime}} z_{z}$ representam a taxa de mudança na produção e na receita líquida quando se faz variação nos limitantes dos volumes de água e doses de nitrogênio. Pode-se dizer que o procedimento numérico implementado para a maximização da produção funciona como segue:

Dado um parâmetro $\mu>0$ e um ponto próximo de $\left(w(\mu), n(\mu), s_{u}(\mu), s_{2}(\mu), z_{u}(\mu), z_{2}(\mu)\right)$ (GONZAGA, 1992), em cada iteração é resolvido aproximadamente o sistema não linear (6)-(11), usando o método de Newton (BERTSEKAS, 2004). Em seguida, é feito decréscimo de $\mu$ e o processo é repetido até que uma condição de parada predeterminada é satisfeita. Da mesma maneira, pode-se implementar um procedimento para a maximização da receita líquida.

Para pesquisar o desempenho dos procedimentos, foram selecionadas as culturas aveia (FRIZZONE et al., 1995) (eq.(12)) e laranja-pera (BERTONHA et al., 1999) (eq.(13)):

$$
\mathrm{p}(\mathrm{w}, \mathrm{n})=3,57510^{-2} \mathrm{w}+1,55410^{-2} \mathrm{n}-5,610^{-5} \mathrm{w}^{2}-5,110^{-5} \mathrm{n}^{2}
$$

em que,

$\mathrm{p}(\mathrm{w}, \mathrm{n})$ - produtividade da aveia, $\mathrm{t} \mathrm{ha}^{-1} ; \mathrm{p}(\mathrm{w}, \mathrm{n})$;

w - lâmina de água aplicada, mm, e

$\mathrm{n}$ - dose de nitrogênio, $\mathrm{kg} \mathrm{ha}^{-1}$.

Os preços para a forragem de aveia, nitrogênio e água foram de $\mathrm{R} \$ 150,00 \mathrm{t}^{-1}, \mathrm{R} \$ 0,50 \mathrm{~kg}^{-1} \mathrm{e}$ $0,020 \mathrm{~m}^{-3}$, respectivamente, $(\mathrm{w}, \mathrm{n}) \in[0,600][0,500]$.

$$
\mathrm{p}(\mathrm{w}, \mathrm{n})=0,306+1,0110^{-2} \mathrm{n}-1,4610^{-5} \mathrm{n}^{2}+4,3510^{-4} \mathrm{w}-4,4710^{-8} \mathrm{w}^{2}
$$

em que,

$\mathrm{p}(\mathrm{w}, \mathrm{n})$ - produtividade de laranja pêra, caixas árvore ${ }^{-1}$;

w - lâmina de água aplicada, L árvore ${ }^{-1}$, e

$\mathrm{n}$ - dose de nitrogênio, $\mathrm{kg}$ árvore ${ }^{-1}$.

$(\mathrm{w}, \mathrm{n}) \in[0,6000][0,5000]$

Na primeira cultura, estudou-se a maximização da produtividade e a maximização da receita líquida. Para a segunda cultura, foi realizada apenas a maximização da produtividade em resposta à aplicação da água e do nitrogênio, motivo pelo qual não foram apresentados os preços normalmente utilizados na maximização da receita líquida.

\section{RESULTADOS E DISCUSSÃO}

Para a cultura da aveia, os valores de produtividade e de receita líquida maximizados, separadamente, após a implementação do MPI em MATLAB, foram de 6,9 $\mathrm{t} \mathrm{ha}^{-1}$, com lâmina de $319,2 \mathrm{~mm} \mathrm{ha}^{-1}$ e dose de nitrogênio de $152,36 \mathrm{~kg} \mathrm{ha}^{-1}$, e de $\mathrm{R} \$ 902,76 \mathrm{ha}^{-1}$, com lâmina de 307,29 $\mathrm{mm} \mathrm{ha}{ }^{-1} \mathrm{e}$ dose de nitrogênio de $119,67 \mathrm{~kg} \mathrm{ha}^{-1}$. Utilizando a metodologia apresentada por FRIZZONE et al. (1995), o valor de produtividade encontrado foi o mesmo, e em relação à receita líquida, pequenas diferenças de $0,64 \%, 0,87 \%$ e $0,27 \%$, respectivamente. É importante ressaltar que, na técnica de linearização por partes (FRIZZONE \& ANDRADE JÚNIOR, 2005), o número de seguimentos em que a função-objetivo é linearizada, influencia no resultado encontrado, e quanto maior esse número, mais próximo da função original se trabalha.

Nas Figuras 1 e 2, mostram-se os resultados de produtividade e de receita líquida, respectivamente, para a cultura da aveia. 


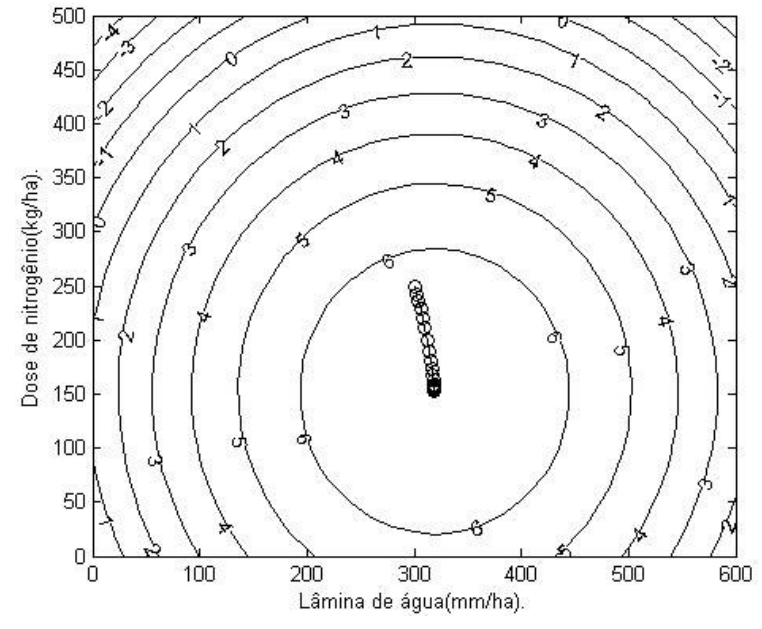

a

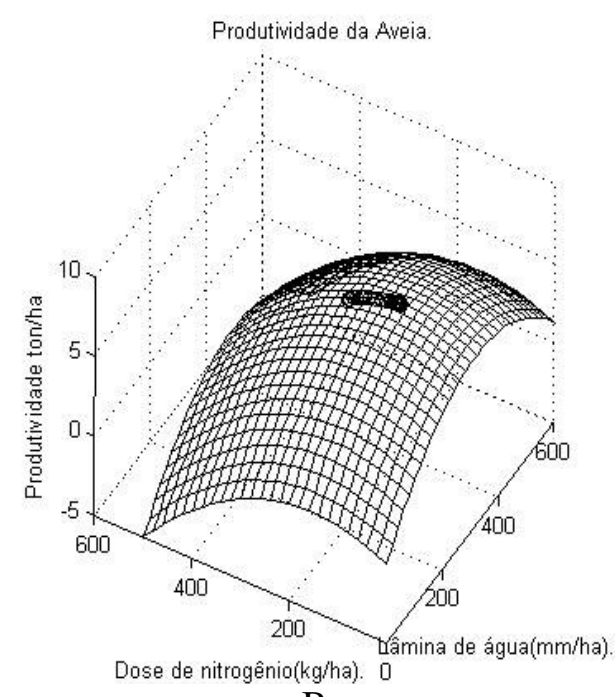

B

FIGURA 1. Curvas de contorno da função $\mathrm{p}(\mathrm{w}, \mathrm{n})$ e trajetória central associada à produtividade (a), e função $p(w, n)$ da aveia (b). Contour curves of the function $\mathbf{p}(\mathbf{w}, \mathbf{n})$ and central trajectory associated with productivity (a) and function $p(w, n)$ of oat $(b)$.

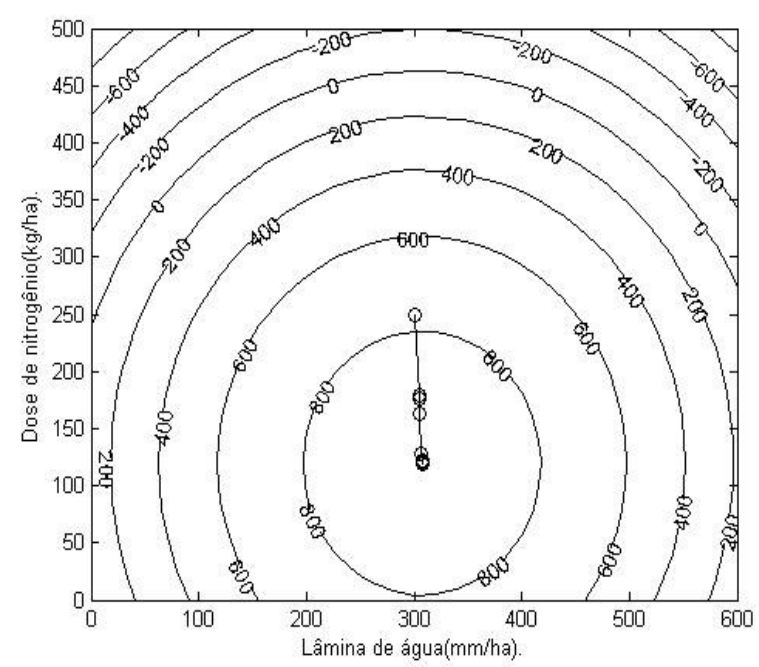

a

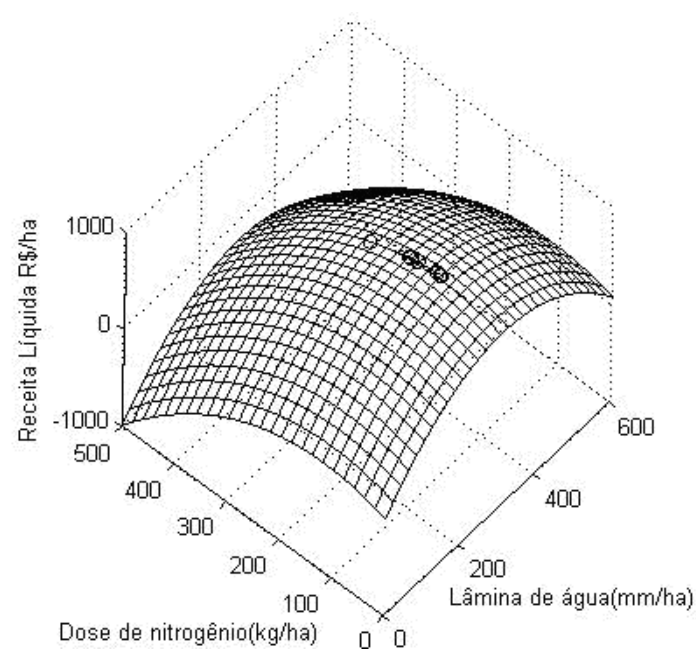

$\mathrm{B}$

FIGURA 2. Curvas de contorno da função $R(w, n)$ e trajetória central associada à renda (a) e função $\mathrm{RL}$ (w, n) da aveia (b). Contour curves of the function $\mathbf{R}(\mathbf{w}, \mathbf{n})$ and central trajectory associated with income (a) RL and function (w, n) of oat (b).

Na Figura 1a, é possível observar pequeno esboço da trajetória central gerada pelos pontos do plano próximos dos pontos $((w(\mu), n(\mu))$ que resolvem aproximadamente a eq.(1), para cada $\mu>0$ fixo, no caso das curvas de contorno de $p(w, n)$. Na Figura 1b, são apresentados os pontos do espaço próximos dos pontos da trajetória central $(w(\mu), n(\mu), p(w(\mu), n(\mu))$ (para cada $\mu>0$ fixo), no caso do gráfico da função $p(w, n)$. A vantagem de utilizar metodologia que segue iterativamente a trajetória central é que permite visualizar a convergência em direção à solução ótima da produtividade quando $\mu$ decresce até tolerância predeterminada. Além disso, a cada iteração, o usuário tem ciência do progresso das soluções aproximadas geradas e da região onde se encontram os valores ótimos dos problemas de produção e da receita líquida, respectivamente. No caso específico deste trabalho, a sequência de pontos gerada pelo MPI que converge à solução ótima é dada por $\left(w^{*}, n^{*}, p\left(w^{*}, n^{*}\right)\right)=(319,29 ; 152,36 ; 6,9)$ (Figura 1b). Além disso, o parâmetro de penalidade $\mu$ permite conhecer, em pequeno número de iterações, a partir de que valor não é 
possível obter melhor valor-objetivo, possibilitando a aplicação parcial do procedimento implementado. Nesta experiência, o $\mu$ em que são alcançados os valores ótimos é $\mu^{*}=1313 e-4$.

Analogamente, nas Figuras $2 \mathrm{a}$ e 2b, são apresentadas as trajetórias centrais associadas às curvas de contorno e gráfico da receita $\mathrm{RL}(\mathrm{w}, \mathrm{n})$, respectivamente. Nesse caso, é possível perceber que a sequência de pontos gerados como soluções aproximadas da eq.(1), convergem para ( $\mathrm{w}^{*}, \mathrm{n}^{*}$, $\left.\mathrm{RL}\left(\mathrm{w}^{*}, \mathrm{n}^{*}\right)\right)=(310 ; 120 ; 902,76)$. Os valores obtidos para as variáveis $s_{l}^{*}, s_{u^{*}}^{*} z_{l}^{*}, z_{\mathrm{u}}^{*}$, na maximização da produção e da receita líquida, respectivamente, são quase zero (ordem $10^{-7}$ ); isso indica que não são significativas as variações ou mudanças nos volumes de água e doses de nitrogênio, respectivamente.

Os resultados da maximização da produtividade referente à laranja-pera estão apresentados nas Figuras 3a e 3b. A produtividade máxima obtida pelo MPI, tipo trajetória central, foi de 3,2 caixas por árvore, com volume de água igual a 5.065,1 L por árvore e dose de nitrogênio igual a $345,9 \mathrm{~kg}$ por árvore.

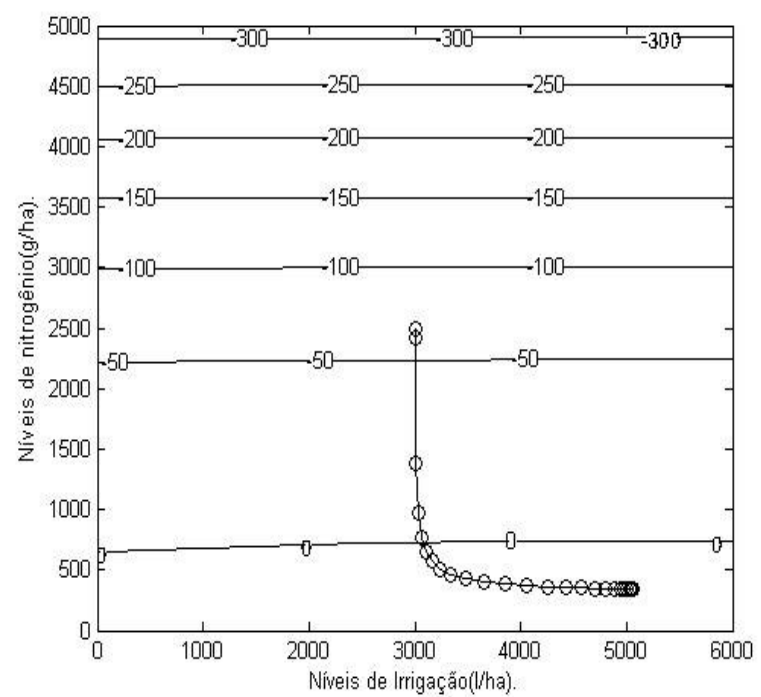

a

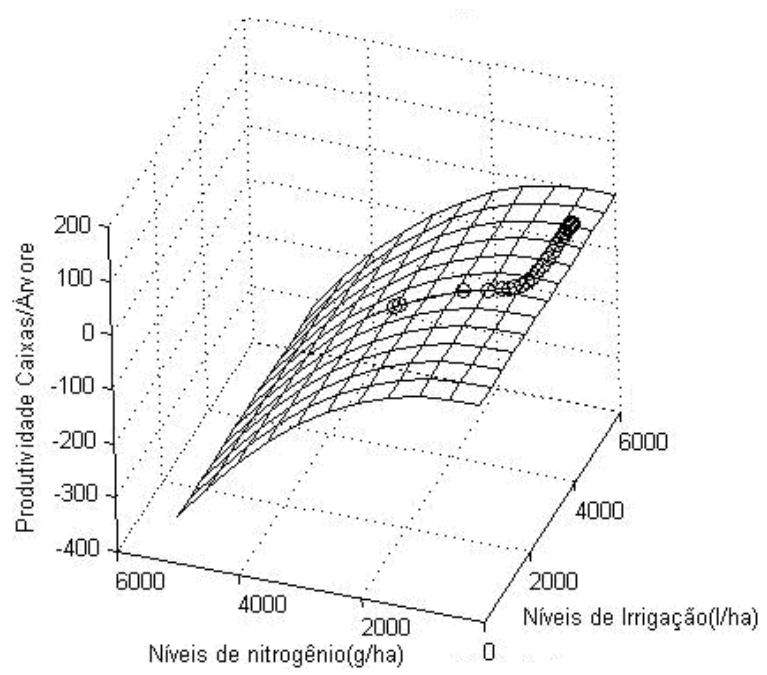

b

FIGURA 3. Curvas de contorno da função $\mathrm{p}(\mathrm{w}, \mathrm{n})$ e trajetória central associada à produtividade (a), e função $p(w, n)$ da laranja-pera (b). Contour curves of the function $\mathbf{p}(\mathbf{w}, \mathbf{n})$ and central trajectory associated with productivity (a) and function $p(w, n)$ of the orange pear (b).

Os resultados apresentados por BERTONHA et al. (1999) foram praticamente os mesmos, com variação apenas no volume de água aplicada por árvore. Da mesma forma, as Figuras mostram a trajetória central gerada pelas soluções ótimas aproximadas de eq.(1), quando da aplicação do MPI, tipo trajetória central. É possível notar que agora a sequência de pontos converge a uma solução ótima dada por $\left(\mathrm{w}^{*}, \mathrm{n}^{*}, \mathrm{p}\left(\mathrm{w}^{*}, \mathrm{n}^{*}\right)\right)=(5.095,6 ; 345,9 ; 3,2)$. Novamente, não parecem significativas as variações dos volumes de água e de doses de nitrogênio, respectivamente.

\section{CONCLUSÕES}

$\mathrm{Na}$ busca de técnicas que trabalham diretamente com a função-objetivo, foi apresentada proposta metodológica implementada em MATLAB 7.1 para maximizar a produção e a receita líquida agrícola separadamente com limitações de insumos (água e fertilizantes), usando o método de pontos interiores (MPI), tipo trajetória central. Com base nos resultados obtidos, foi possível concluir que a metolodologia apresentada constitui alternativa confiável, considerando os resultados semelhantes aos publicados na literatura, utilizando-se de programação separável para a resolução dos problemas de maximização da produção e da receita líquida em cenários hidroagrícolas. 


\section{REFERÊNCIAS}

AGUIAR, J.V. A função de produção na agricultura irrigada. Fortaleza: Imprensa Universitária, 2005. $196 \mathrm{p}$.

BAIO, H.R.F.; ANTUNIASSI, U.R.; BALASTREIRE, L.A.; CAIXETA FILHO, J.V. Modelo de programação linear para seleção de pulverizadores agrícolas de barras. Engenharia Agrícola, Jaboticabal, v.24, n.2, p. 355-363, 2004.

BARBOZA, C.B.; OLIVEIRA, A.R.L. Planejamento do tratamento por radioterapia através de métodos de pontos interiores. Pesquisa Operacional, Rio de Janeiro, v.26, n.1, p.1-24, 2006.

BERTONHA, A.; FRIZZONE, J.A.; MARTINS, E.N. Irrigação e adubação nitrogenada na produção de laranja-pera. Acta Scientiarum, Maringá, v.21, n.3, p.537-542, 1999.

BERTSEKAS, D.P. Nonlinear Programming. Belmont: Ed. Athena Scientific, 2004. 780 p.

FRIZZONE, J.A.; ANDRADE JÚNIOR, A.S. Planejamento de irrigação: análise de decisão de investimento. Brasília: Embrapa Informação Tecnológica, 2005. 626 p.

FRIZZONE, J.A.; TEODORO, R.E.F.; PEREIRA A.S.; BOTREL, T.A. Lâmina de água e doses de nitrogênio na produção de aveia (Avena sativa L.) para forragem. Scientia Agricola, Piracicaba, v.52, n.3, p.578-586, 1995.

GONZAGA, C.C. Path following methods for linear programming. SIAM Review, Ann Arbor, v.34, n.2, p.167-227, 1992.

KARMARKAR, N. A new polynomial-time algorithm for linear programming. Combinatorica, Berlin, v.4, n.2, p.373-395, 1984.

OJIMA, O.R.L.A.; YAMAKAMI, A. Modelo de programação quadrática para análise da movimentação logística e comercialização da soja brasileira. Engenharia Agrícola, Jaboticabal, v.26, n.2, p.552-560, 2006.

PEREIRA, O.C.N.; BERTONHA, A.; FREITAS, P.S.L. de; GONÇALVES, A.C.A.; REZENDE, R.; SILVA, F.A. da. Produção de alface em função de água e nitrogênio. Acta Scientiarum Agronomy, Maringá, v.25, n.2, p.381-387, 2003. 